\title{
ARTICLE \\ RTL Implementation of White Balance Algorithm Based on ZYNQ
}

\section{Jie Zhou*, Keyao Li}

Chengdu University of Technology, Chengdu, Sichuan, 610059, China

\begin{tabular}{l}
\hline ARTICLE INFO \\
\hline Article history \\
Received: 8 August 2019 \\
Revised: 9 August 2019 \\
Accepted: 10 October 2019 \\
Published Online: 16 October 2019 \\
\hline Keywords: \\
White balance \\
ZYNQ \\
RTL \\
Gray world
\end{tabular}

\section{Introduction}

$\mathrm{W}$ hite balance is a very important concept in the field of television camera. White balance is produced with the reproduction of color reality in electronic images. White balance was applied earlier in the field of professional photography, and is now widely used in household electronic products (household video camera, digital camera). The white balance algorithm includes manual white balance and automatic white balance. This paper briefly introduces the automatic white balance algorithm and the ZYNQ implementation of the algorithm.

\section{Key Technical Analysis}

\begin{abstract}
In view of the common automatic white balance algorithm complexity is too high and the characteristics of the hardware to realize real-time. This paper combines gray world model and the advantage of white point detection algorithm, an adaptive control process is used to calculate gain coefficient and to picture the three-component white balance correction. On this basis to realize the automatic white balance based on the ZYNQ RTL implementation of the algorithm. Compared with the traditional method, the cost of hardware is reduced, and the efficiency and flexibility of the algorithm are improved. Experimental results show that the algorithm can run smoothly and realize accurate correction of off-color images.
\end{abstract}

\subsection{ZYNQ}

The ZYNQ-7000 scalable processing platform is the latest in a series of products using the same $28 \mathrm{~nm}$ programmable technology as the new generation of syringes fpgas (artix-7 and kintex-7fpga). Programmable logic can be configured by the user and connected together through "interconnect" modules, which can provide user-defined arbitrary logic functions to extend the performance and functionality of the processing system. However, unlike fpgas with embedded processors, the ZYNQ-7000 family of processing systems can not only be started at boot time, but can also be configured with programmable logic as needed. With this approach, the software programming model is identical to the full-featured standard ARM

\footnotetext{
*Corresponding Author:

Jie Zhou (1995-),

Male, a native of Luzhou in Sichuan Province, China, master candidate in Chengdu University of Technology;

Correspondence address: Chengdu University of Technology, No. 1 East 3rd Road, Erxianqiao, Chenghua District, Chengdu, Sichuan, 610059, China;

E-mail:3330491414@QQ.com
} 
approach to SoC. The white balance algorithm can be realized efficiently and quickly by using the advantages of ARM and FPGA.

\subsection{Constancy of the Color}

The color is not an actual thing; it is just the result of our brain and retina processing. One of the special functions of our visual system is to be able to determine the true color of an object even when the light source changes, a robustness that computers and other processing machines do not have. Color constancy, also can be understood simply, under the circumstance that outside illuminant changes, still can retain the object original color. We study color constancy and come up with algorithms that aim to make computers have this special function of the human vision system, restoring the original color of an image under different light sources. According to the sensory judgment of human eyes, when the color temperature is 6000 $\mathrm{K}$ (kelvin), we evaluate that the color of the image we see is white, while in other color temperatures, the image we see is biased. We hope to correct this display phenomenon called white balance through the algorithm.

\subsection{Grayscale World Algorithm}

In Marc Ebner's monograph Color Constancy, Gray World algorithm of white balance is proposed. The grayscale world algorithm is based on the grayscale hypothesis, which holds that: for an image with a large number of color changes, the average value of the three-color components $\mathrm{R}, \mathrm{G}$ and $\mathrm{B}$ will tend to the same grayscale value. In the physical sense, the grey-world method assumes that the mean value of the average reflection of natural objects to light is, on the whole, a definite value, which is approximately "grey". The white balance algorithm applies this assumption to the image to be processed, which can eliminate the influence of ambient light from the image and obtain the original scene image.

Suppose the average value of the three color components $\mathrm{R}, \mathrm{G}$ and $\mathrm{B}$ approaches to $\mathrm{X}$ :

$$
\mathrm{K}=\left(\mathrm{R}_{\mathrm{ver}}+\mathrm{G}_{\mathrm{ver}}+\mathrm{B}_{\mathrm{ver}}\right) / 3
$$

Where $R_{\text {ver, }}, G_{v e r}$ and $B_{\text {ver }}$ represent the mean values of red, green and blue color channels respectively. The gain of channel $\mathrm{R}, \mathrm{G}$ and $\mathrm{B}$ is defined as $\mathrm{Xr}, \mathrm{Xg}$ and $\mathrm{Xb}$ respectively:

$$
\begin{aligned}
& \mathrm{X}_{\mathrm{r}}=\mathrm{X} / \mathrm{R}_{\mathrm{ver}} \\
& \mathrm{X}_{\mathrm{g}}=\mathrm{X} / \mathrm{G}_{\mathrm{ver}} \\
& \mathrm{X}_{\mathrm{b}}=\mathrm{X} / \mathrm{B}_{\mathrm{ver}}
\end{aligned}
$$

According to the Von Kries diagonal model, it is assumed that the updated R,G,B are Rn,Gn,Bn:

$$
\begin{aligned}
& {\left[\begin{array}{l}
\mathrm{RN} \\
\mathrm{Gn} \\
\mathrm{Bn}
\end{array}\right]=\left[\begin{array}{ccc}
\mathrm{Xr} & 0 & 0 \\
0 & \mathrm{Xg} & 0 \\
0 & 0 & \mathrm{Xb}
\end{array}\right]\left[\begin{array}{l}
\mathrm{R} \\
\mathrm{G} \\
\mathrm{B}
\end{array}\right]} \\
& \mathrm{Rn}=\mathrm{Xr} * \mathrm{R} \\
& \mathrm{Gn}=\mathrm{Xg} \mathrm{g}^{*} \mathrm{G} \\
& \mathrm{Bn}=\mathrm{Xb} \mathrm{b}^{*} \mathrm{~B}
\end{aligned}
$$

\subsection{The color difference detection}

In order to detect the chromatic aberration in the image more accurately, the original image is converted to $\mathrm{Y} \mathrm{Cb}$ $\mathrm{C} \mathrm{r}$ space, which has a similar constitution principle to human visual perception process, and the brightness information is separated from the chroma information, which is less affected by the brightness change and is widely applied in various fields. The transformation relation of mapping from $\mathrm{RGB}$ space to $\mathrm{Y} \mathrm{Cb} \mathrm{Cr}$ space is as follows:

$$
\left[\begin{array}{c}
Y \\
C b \\
C r
\end{array}\right]=\left[\begin{array}{ccc}
0.2990 & 0.5870 & 0.1440 \\
-0.1687 & -0.3313 & 0.5000 \\
0.5000 & -0.4187 & -0.0813
\end{array}\right]\left[\begin{array}{l}
R \\
G \\
B
\end{array}\right]
$$

Blue-green difference and red-green difference $(\mathrm{Cb}$ and $\mathrm{Cr}$ ), white difference is 0 , where $\mathrm{Y}$ represents the brightness of the image (luma).

Nakano proposed the following constraint conditions according to the characteristics of white equilibrium:

$$
\left\{\begin{array}{c}
Y>\mathrm{X} \\
-a<C b<a \\
-b<C r<b
\end{array}\right.
$$

The pixels within the constraint area are considered white, while the pixels outside are considered non-white. The average chromatic aberration of white pixels is calculated to replace the chromatic aberration of the whole image, thus improving the accuracy of color temperature estimation and reducing the complexity.

\section{Hardware Architecture Design}

The following hardware architecture is designed to implement and verify the white balance algorithm.

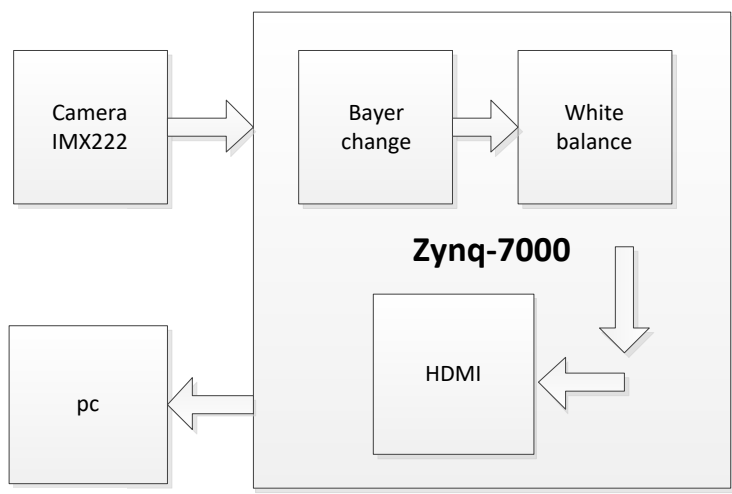

Figure1. Hardware system architecture 
As shown in the figure, firstly, SONY's IMX222 camera is used to collect image data and pass the data to Bayer module for image conversion, and then the image data is passed to the core white balance module for white balance calculation. Then through the HDMI conversion module after the image conversion output display.

\subsection{Bayer Change Module}

Since the image data output by Camera IMX222 is in Bayer image format, white balance calculation cannot be performed directly. Therefore, we need to convert Bayer image format to RGB image format before calculating white balance. Bayer is the original image format inside the camera, commonly suffixed.raw. Our cameras take pictures and store them on a memory card in.jpeg or whatever format, and they're converted from.raw. As shown in the figure below, the bayer color filter array is composed of half $\mathrm{G}, 1 / 4 \mathrm{R}$ and $1 / 4 \mathrm{~B}$.

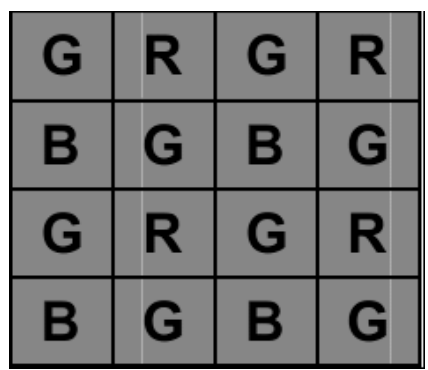

Figure 2 Bayer color filter array

Each pixel contains only a portion of the spectrum and must be interpolated to achieve the RGB value for each pixel. To get the RGB format for each pixel from the Bayer format, we need to fill in the missing two colors by interpolation. There are many interpolation methods (including domain, linearity, $3 * 3$, etc.), speed and quality tradeoff, this paper chooses the $3 * 3$ matrix method.

\subsection{White Balance Module}

In this module, the use of grayscale world algorithm will lead to division in the calculation of white balance algorithm. In ZYNQ, although there is a dedicated division IP, it will consume a lot of resources. Therefore, the method of using displacement instead of division proposed in this paper will greatly save resources. The images collected by the camera IMX222 in this system are 1080*1920. Therefore,

the total number of bits processed is:

$\mathrm{Z}=(1080 * 1920)_{10}$

$=(1 \mathrm{FA} 400)_{16}=(111111010010000000000)_{2}$

Make an approximation here:

Znew $=(200000)_{16}$
Then the approximate error:

$\mathrm{W}=($ Znew-Z $) / \mathrm{Z}=1.13 \%$

Therefore, the sum and low 21 in the white balance algorithm can be removed and the high value can be retained, which greatly reduces the complexity of the algorithm implementation under the condition of low error.

\section{Test Results Presentation}

The field test of the system shows that the IMX222 camera can collect image data smoothly, the algorithm can run in the system perfectly for a long time, and the white balance correction of the image can be realized. As shown in Figure 3 and Figure 4 below, figure 3 is the unprocessed picture and figure 4 is the picture processed by the system.

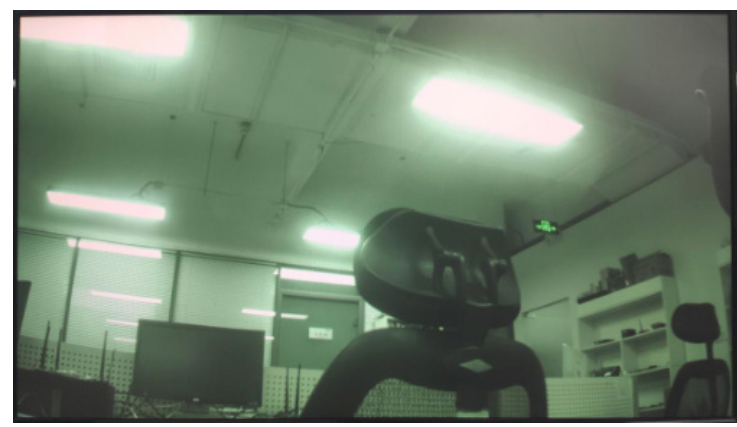

Figure 3. Unprocessed image

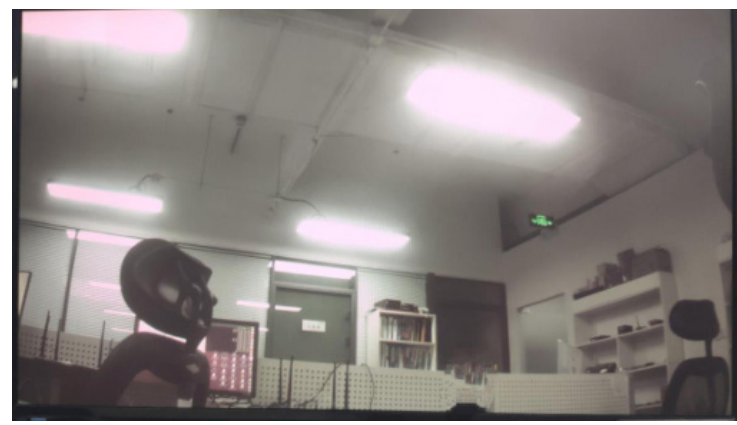

Figure 4. Processed image

\section{Conclusion}

In this paper, aiming at the characteristics of high complexity of automatic white balance algorithm and poor real-time performance of hardware implementation, the advantages of grayscale world model and white point detection algorithm are integrated. On ZYNQ-7000, the white balance algorithm is realized by using the powerful computing power and design platform of ZYNQ. Moreover, the approximate advantages of some algorithms are used to simplify the consumption of hardware resources and improve the efficiency and timeliness of the algorithm. After testing, the whole system can run smoothly on ZYNQ-7000 and realize accurate correction of off-col- 
or images.

\section{References}

[1] JarnoNikkanen, Tampere(FI).Image Processing Device with Au-tomatic White Balance[P]. US:2009/0295938, 2006-08-28.

[2] Ramanath R,Drew M S. von Kries Hypothesis[J]. Computer Vision, 2014:874-875.

[3] Aoide N. Low-voltage Differential Signaling[M]. Soph Press, 2013.

[4] Silva V D,Chesnokov V,Larkin D.A Novel Adaptive Shading Correction Algorithm for Camera Systems[J]. Electronic Imaging,2016,2016(18):1-5.

[5] Lu peilong, fu yide. Design and implementation of image preprocessing algorithm on ZYNQ chip[J]. Computer and digital engineering, 2015(6):1118-1120.

[6] Yin weiwei. Image color deviation detection method based on $\mathrm{YCbCr}$ color space[J]. Computer Era, 2017(9):47-50.

[7] Jin huangbin, qin xing, guo binlin. Automatic white balance algorithm based on grayscale world and white point detection[J]. Electronic devices 2011.04.
[8] BarnardK.Practical Color Constancy[D]. PhD ThesisSimonFra-ser University, School of Computing, 1999.01.

[9] Yang Z T,Ruan P,Zhai B.Auto-exposure Algorithm for Scenes with High Dynamic Range Based on Image Entropy[J]. Acta Photonica Sinica, 2013, 42(6):742-746.

[10] Barnard K,Cardei V,Funt B. A comparison of computational color constancy algorithms.I: Methodology and experiments with synthesized data[M]. IEEE Press,2002.

[11] Lam EY. Combining gray world and retinex theory for automatic white balance in digital photography[C]. International Symposium on Consumer Electronics. IEEE, 2005.

[12] Gasparini F,Schettini R. Color balancing of digital photos using simple image statistics[J]. Pattern Recognition,2004,37(6):1201-1217.

[13] Weng C C, Chen H,Fuh C S. A novel automatic white balance method for digital still Cameras[C] IEEE International Symposium on Circuits and Systems. IEEE Xplore, 2006. 